\title{
Endoscopic injection sclerotherapy for bleeding varices in children with intrahepatic and extrahepatic portal venous obstruction: Benefit of injection tract embolisation
}

\author{
V L Bandika, E A Goddard, R De Lacey, R A Brown
}

Background. The outcome of sclerotherapy for bleeding oesophageal varices may be influenced by injection technique. In a previous study at our institution, sclerotherapy was associated with a high re-bleeding rate and oesophageal ulceration. Embolisation of the injection tract was introduced in an attempt to reduce injectionrelated complications.

Methods. To determine the outcome and effectiveness of injection tract embolisation in reducing injection-related complications, we retrospectively reviewed a series of 59 children who underwent injection sclerotherapy for oesophageal varices (29 for extrahepatic portal vein obstruction (EHPVO) and 30 for intrahepatic disease) in our centre.

Results. Sclerotherapy resulted in variceal eradication in only $11.8 \%$ of the children (mean follow-up duration: 38.4 months).
Variceal eradication with sclerotherapy alone was achieved in $20.7 \%$ and $3.3 \%$ of EHPVO and intrahepatic disease patients, respectively. Injection tract embolisation was successful in reducing the number of complications and re-bleeding rates. Complications that arose included: transient pyrexia (16.7\%); deep oesophageal ulcers (6.7\%); stricture formation (3.3\%); and re-bleeding before variceal sclerosis (23\%).

Conclusion. Injection sclerotherapy did not eradicate oesophageal varices in most children. Injection tract embolisation by sclerosant was associated with fewer complications and reduced re-bleeding rates.

S Afr Med J 2012;102(11):884-887. DOI:10.7196/SAMJ.6263
Portal hypertension due to intrahepatic disease or extrahepatic portal vein obstruction (EHPVO) is an important cause of upper gastrointestinal bleeding in children. About $50 \%$ of children with EHPVO present with bleeding from oesophageal varices..$^{1-3}$ Improvements in the management of children with intrahepatic disease have led to increased survival, consequently contributing to the long-term burden of portal hypertension. ${ }^{1,3-5}$

The peak age of variceal bleeding, although not clearly defined, relates to a critical point where wall tension exceeds variceal wall strength. Other determinants of a 'herald' bleed include upper respiratory infection, fever and aspirin ingestion. ${ }^{6}$ These factors directly or indirectly increase portal venous pressure or result in fever-related tachycardia which increases cardiac output.

Since its introduction, endoscopic sclerotherapy of bleeding oesophageal varices, where variceal banding is not feasible, has remained the mainstay treatment for haemodynamically stable patients and where clotting abnormalities have been corrected. Concerns about its efficacy and safety in the management of oesophageal varices in children and adults have recently been raised. Several innovations have been aimed at improving sclerotherapy outcome. We prefer paravariceal injection with injection tract embolisation. Intravariceal injection has also been used. Ethanolamine oleate is our preferred sclerosant. However, different types of sclerosants have been used without affecting outcome.

School of Child and Adolescent Health, Red Cross War Memorial Children's Hospital and University of Cape Town, Cape Town

V L Bandika, MB ChB, MMed (Paed)

E A Goddard, MSc (Med), MB ChB, MMed (Paed), $\mathrm{PhD}$

R De Lacey, MB ChB, FCP (Paed)

R A Brown, MB ChB, FRCS (Edin), FCS (SA), MPhil (UStell)

Corresponding author: VL Bandika (bandikav@yahoo.com)
Progress has been made in developing newer endoscopic techniques for managing bleeding oesophageal varices which are safer, but pose challenges in terms of availability, cost and operative techniques. Endoscopic variceal band ligation is safer than sclerotherapy, but the diameter of the band applicator makes it impossible to insert the applicator into the small oesophagus of children aged $<3$ years. ${ }^{7}$ Coupled with the success and increased availability of liver transplantation (LT), the successful management of bleeding oesophageal varices in these children is required in preparation for LT or other appropriate surgery.

We aimed to assess whether the use of injection tract embolisation improves the efficacy and safety of sclerotherapy compared with the previously used direct injection method in the management of oesophageal varices where banding is not feasible. We retrospectively evaluated a series of children with bleeding oesophageal varices, treated with sclerotherapy in our centre. We analysed the outcome of endoscopic sclerotherapy performed with injection tract embolisation and its relationship to the underlying aetiology.

\section{Methods}

Between 1998 and 2007, 70 children who presented with bleeding oesophageal varices secondary to portal hypertension (41 with intrahepatic conditions and 29 with EHPVO) underwent injection sclerotherapy at Red Cross War Memorial Children's Hospital. The patients presented with upper gastro-intestinal bleeding manifested as haematemesis or malaena. Resuscitation and stabilisation using intravenous fluids, blood, platelets and fresh-frozen plasma transfusions, was performed if required. An infusion of octreotide was administered at $1-5 \mu \mathrm{g} / \mathrm{kg}$ in a $5 \%$ dextrose solution at $5 \mathrm{ml} /$ hour, in decreasing doses over $4-5$ days. If these measures failed to arrest upper gastro-intestinal bleeding, emergency endoscopy was performed. Failure to arrest bleeding by sclerotherapy was managed by placement of a Sengstaken Blakemore tube (SSBT).

Once stable, patients underwent elective videoscope endoscopy under general anaesthesia. Careful endoscopic assessment of the foregut was performed to identify the site and true cause of bleeding. 
Patients with non-variceal upper gastro-intestinal bleeding were excluded from analysis.

The extent of oesophageal varices and the presence of gastric varices were documented; $5 \%$ ethanolamine oleate was injected as a sclerosant, usually $0.5-0.75 \mathrm{ml}$ per injection, at multiple (up to 5) sites paravariceally - enough to produce a visible blanch with mucosal swelling. Injection tract embolisation was performed by continuation of the sclerosant injection using the terminal $0.2 \mathrm{ml}$ as the needle was withdrawn. The number of injections and amount of sclerosant used were noted. Subjects were admitted to a high-care unit following sclerotherapy and monitored for adverse effects. Repeat sclerotherapy was performed at 2-weekly intervals until identified varices were eliminated. Because the recurrence of varices depends on the underlying pathogenesis, further investigations were undertaken to identify the underlying cause of portal hypertension.

\section{Results}

The study population comprised 70 children: 29 with EHPVO and 41 with intrahepatic causes of portal hypertension. Eleven children with intrahepatic disease secondary to biliary atresia were not included in the analysis of sclerotherapy details due to missing clinical data, but were included in the analysis of outcome.

Intrahepatic causes of portal hypertension included: biliary atresia (20); congenital hepatic fibrosis syndromes (3); neonatal hepatitis (1); auto-immune hepatitis (1); Alagille syndrome (1); Langerhans cell histiocytosis (1); and idiopathic cirrhosis (3). Clinico-demographic details are summarised in Table 1.

Regardless of the cause of portal hypertension, all patients had severe enough upper gastro-intestinal bleeding to necessitate a blood transfusion. Mean patient age at first sclerotherapy, presumed to coincide with the age at first episode of upper gastro-intestinal bleeding, was 3 years and 7 months.

Sclerotherapy and octreotide were effective in arresting acute variceal bleeding in 56/59 (94\%) patients. SSBT insertion was used in 3 patients, and endoscopic variceal band ligation (EVBL) was performed in 4 of the older patients. No emergency definitive surgical procedure was offered.

Gastric varices were identified in 22/59 (37.3\%) patients - slightly more than in the EHPVO group, but not statistically significant. Surprisingly, gastric varices were rarely found to be a cause of upper gastro-intestinal bleeding; no patient had any additional procedure to specifically address bleeding varices in the gastric region of the oesophageal gastric junction. Of interest is that the presence of gastric varices was used as an indication for selecting patients for shunt surgery in EHPVO.

Among children in the intrahepatic disease group, 53.3\% had initial or first oesophageal bleeding before age 2 years, compared with $37.9 \%$ in the EHPVO group, although this difference was not statistically significant. No significant difference in the number of patients presenting with first variceal bleeding was noted within all the age categories in both groups $(p>0.05)$. In the intrahepatic group, the distribution pattern of age at first variceal bleeding was related to the nature of the underlying disease, modification effect of therapy offered and subsequent course of the disease spectrum.

Sclerotherapy alone was successful in controlling the bleeding from oesophageal varices in 7 (11.9\%) patients. However, 2 of these patients with EHPVO presented as re-bleeders after previous variceal sclerosis and were managed by additional sclerotherapy sessions.

Eighteen of 29 (62.1\%) EHPVO patients were offered surgical shunts. Meso-Rex shunts were the most favoured, performed on 8

Table 1. Clinico-demographic details of patients undergoing sclerotherapy

\begin{tabular}{lll}
\hline & $\begin{array}{l}\text { EHPVO } \\
(\boldsymbol{N = 2 9 )}\end{array}$ & $\begin{array}{l}\text { Intrahepatic disease } \\
(\boldsymbol{N = 3 0})\end{array}$ \\
\hline Gender (male:female) & $20: 9$ & $10: 20$ \\
Age at first sclerotherapy (months), mean (range) & $42.6(9-112)$ & $43.0(7-159)$ \\
Number of sclerotherapy sessions, mean & 6.48 & 3.73 \\
$\quad$ Median (range) & $5(1-16)$ & $3(1-11)$ \\
Number of injections to variceal sclerosis, mean & 25.46 & 16.70 \\
$\quad$ Median (range) & $22(3-59)$ & $13(3-52)$ \\
SSBT & 0 & 3 \\
Co-existent gastric varices & 13 & 9 \\
EHPVO = extrahepatic portal vein obstruction; SSBT = Sengstaken Blakemore tube. & &
\end{tabular}

Table 2. Complications of the different sclerotherapy injection techniques

\begin{tabular}{|c|c|c|}
\hline & \multicolumn{2}{|c|}{ Study } \\
\hline & $\begin{array}{l}\text { Hill and Bowie }^{8} \text { (no injection tract } \\
\text { embolisation) }(N=33) \\
n(\%)\end{array}$ & $\begin{array}{l}\text { Current (injection tract embolisation, } \\
\text { number of complication occurrence/total } \\
\text { sclerotherapy sessions) }(N=300) \\
n(\%)\end{array}$ \\
\hline Transient pyrexia & $13(39)$ & $50(16.7)$ \\
\hline Deep oesophageal ulcers & $10(30)$ & $20(6.7)$ \\
\hline Stricture & $4(12)$ & $10(3.3)$ \\
\hline Re-bleeding before variceal sclerosis & $12(36)$ & $70(23)$ \\
\hline Confirmed sepsis & - & $16(5.3 \%)$ \\
\hline
\end{tabular}


patients, 2 of whom were post LT. Splenorenal and mesocaval shunts were offered to 6 and 4 patients, respectively.

Eleven patients underwent LT. No definitive procedure was offered to 7 patients who were awaiting donor availability or had a condition excluding them from LT at that time. Nine patients were lost to follow-up. Four deaths were reported, occurring during admission for sclerotherapy; cause of death was not directly related to sclerotherapy or its complications, but to the underlying disease.

Injection tract embolisation was associated with a lower complication rate (Table 2). A greater number of confirmed sepsis cases were reported among intrahepatic disease patients (17/30 (56.7\%) compared with 9/29 (31\%) EHPVO patients; $p=0.067$ ). Organisms cultured included Escherichia coli, Staphylococcus epidermidis, Klebsiella species and Acinetobacter species.

\section{Discussion}

The study cohort had a nearly even distribution of EHPVO and intrahepatic disease as a cause of varices, allowing us to compare modes of sclerotherapy and determine if outcome was influenced by underlying aetiology.

Early portoenterostomy and LT availability have contributed to a higher incidence of children requiring sclerotherapy for intrahepatic portal hypertension compared with the Hill and Bowie ${ }^{8}$ study where most patients had EHPVO.

The age of first variceal bleeding in the intrahepatic group was determined by the underlying disease process. In this study, more children with intrahepatic disease than EHPVO bled within the first 2 years of life. The reason for this is the contribution of a delayed diagnosis of biliary atresia in these children. Three patterns of intrahepatic variceal bleeding were also demonstrated: first group (0 24 months) - missed biliary atresia; second group (25 - 60 months) mainly attributable to poorly functioning or failed portoenterostomy; third group (5 - 9 years) - could be explained as a critical point where intrahepatic cholangiopathy effect is maximal and overrides biliary drainage offered by portoenterostomy, and where LT should be considered in children with previously 'successful' portoenterostomy. In EHPVO, there was an equal distribution of patients in the first 5 years of life, suggesting an age-independent aetiology.

The results of our study do not support other reports showing sclerotherapy to be successful in both groups in eradicating oesophageal varices (i.e. controlling bleeding without a need for a definitive procedure) ${ }^{9,10}$ Only $11.8 \%$ of patients showed variceal eradication on sclerotherapy alone after a mean follow-up duration of 38.4 months. In contrast to other studies, ${ }^{2,9,10}$ the success rate with sclerotherapy alone on EHPVO was $20.7 \%$, which was low. Most children in the successful group presented just before puberty and were able to re-canalise or spontaneously achieve adequate portosystemic collaterisation (thought to be hormonally induced), which could have contributed to this observation. This was possibly the basis of previous studies which reported success.

Anecdotal reports have come from parents, teachers and paediatricians about the resolution of behavioural and cognitive problems after physiological surgical shunts and the low success rate after sclerotherapy. Meso-Rex shunt as a definitive procedure was recommended in this group and was offered in $35.8 \%$ of patients, of whom $6.5 \%$ were post LT with associated portal vein obstruction. There is, to date, no consensus on EHPVO management. Some studies advocate Meso-Rex shunt in all children with portal vein thrombosis and cavernous transformation. ${ }^{11}$ Early age at first presentation, the presence of fundal varices which are usually not easily amenable to sclerotherapy as shown in a previous study in the same setting, ${ }^{12}$ life-threatening bleeds, numerous sclerotherapy sessions and failure to thrive could also be considered as indications for Meso-Rex shunt. Likewise, LT is the only definitive treatment in biliary atresia with a failed Kasai portoenterostomy.

In this series, $44.8 \%$ of EHPVO and $30 \%$ of intrahepatic disease patients had gastric varices. We also demonstrated primary co-existence of both oesophageal and gastric varices rather than the secondary opening of gastric collaterals after oesophageal variceal sclerosis. However, on endoscopy, no corresponding gastric variceal bleeding was noted in either group. The oesophageal variceal bleeding could possibly act as a 'sump', reducing intravariceal pressures in the gastric venous system.

The largest contributor to intrahepatic portal hypertension was biliary atresia, which ultimately necessitated LT. Sclerotherapy was used to 'buy time' while awaiting LT, enabling 11/30 children to undergo LT. Endoscopic variceal band ligation has been shown to be superior due to fewer complications, ${ }^{13,14}$ but its use in children aged $<3$ years remains technically difficult due to the diameter of the smallest band applicator. As in our scenario, where $53.3 \%$ of intrahepatic disease patients and $41.4 \%$ with EHPVO presented with variceal bleeding were aged $<3$ years, sclerotherapy and octreotide have to be used as a first treatment option and subsequently sclerotherapy alone in controlling variceal bleeding while awaiting LT or an appropriate shunt procedure in EHPVO. Recently, Duche et al..${ }^{15}$ demonstrated the effectiveness and safety of prophylactic sclerotherapy and octreotide in controlling oesophageal varices in biliary atresia.

A combination of medical management using octreotide infusion and sclerotherapy was effective in arresting variceal bleeding in both groups in our study. Only 3/59 (5.1\%) children required SSBT placement to arrest variceal bleeding, supporting previous findings. ${ }^{2,16}$

As in other studies, ${ }^{16,17} 3$ - 5 sessions of sclerotherapy at 2-weekly intervals were needed to achieve variceal sclerosis. In 7 children who showed variceal eradication with sclerotherapy alone, an additional 2 sessions were required. The amount of sclerosant to cause visible blanch was $0.7 \mathrm{ml} /$ injection, similar to other studies within the range of $0.5-1 \mathrm{ml} /$ injection. ${ }^{2}$ The mean number of injections per session was 4.27 , with a mean of $2.98 \mathrm{ml}$ sclerosant administered per session.

Injection tract embolisation with sclerosant was successful in the overall reduction of complications. Hill and Bowie ${ }^{8}$ expressed complication rates as a one-time event which could have overestimated the true occurrence. We appreciate the difficulties in comparison due to difference in presentation of complication rates. Transient pyrexia, stricture, ulcers and re-bleeding before variceal sclerosis were less incident in our study compared with non-tract embolisation in the hospital's previous series (Table 2). ${ }^{8}$ While the authors of the comparative study attributed the high re-bleeding rates to a high rate of gastric varices, we demonstrated similar high gastric variceal rates but these varices were not implicated as a cause of bleeding. Our high re-bleeding rates might have been due to slough bleeding which was erroneously labelled as re-bleeding. Our observed oesophageal stricture rate of $3.3 \%$ was lower than in other studies. ${ }^{8,16,17}$ No statistically significant difference was noted in endoscopy-associated bloodstream infections in either group, but over $50 \%$ of patients in the intrahepatic group had sepsis compared with $37 \%$ in the EHPVO group. This could be due to a probable bias in selecting patients in the intrahepatic group. The patients with significant liver dysfunction on presentation were not offered sclerotherapy, especially if they were not eligible for LT. Furthermore, more patients in this group died from primary liver disease or were lost to follow-up. The net effect was the selection of patients with portal hypertension from intrinsic liver disease, but without a significant liver dysfunction impairing immunity.

Sclerotherapy was not associated with any mortality. However, some patients in the intrahepatic group died from the primary 


\section{RESEARCH}

disease before definitive treatment was offered. The higher number of patients lost to follow-up in the intrahepatic group could have been due to patients in this group who were not eligible for LT, and either died at home or sought care from other health facilities.

In conclusion, a similar outcome of sclerotherapy was demonstrated in both groups in our study. Sclerotherapy alone was unsuccessful in controlling oesophageal varices in both groups. However, it was useful in managing acute variceal bleeding and should be offered as an option, particularly in the young patient where endoscopic variceal banding is not technically possible. We now routinely employ injection tract embolisation by sclerosant, which has greatly contributed to reducing sclerotherapy complications.

Acknowledgements. We acknowledge the staff of the Red Cross War Memorial Children's Hospital for their invaluable contribution to this work

\section{References}

1. Renard TH, Andrew WS, Rollins N, et al. Use of distal spleno-renal shunt in children referred fo liver evaluation. J Pediatr Surg 1994;29:403-406. [http://dx.doi.org/10.1016/0022-3468(94)90579-7] 2. Stringer MD, Howard ER. Long term outcome after injection sclerotherapy for oesophageal varices in children with extra-hepatic portal hypertension. Gut 1994;35:57-59. [http://dx.doi.org/10.1136/ gut.35.2.257

3. Alonso EM, Hackworth C, Whitington PF. Portal hypertension in children. Clin Liver Dis 1997;1:201-222. [http://dx.doi.org/10.1016/51089-3261(05)70264-6]
4. Howard ER, Stomatakis ID, Mowat AP. Management of oesophageal varices in children by injection sclerotherapy. J Pediatr Surg 1984;19:2-5. [http://dx.doi.org/10.1016/50022-3468(84)80003-2] . Ebru Y, Haluk E, Gonca T, et al. Oesophageal variceal bleeding secondary to portal hypertension Endoscopic sclerotherapy as first step treatment. [http://dx.doi.org/10.1089/10926420260188100]

6. Spence RA, Johnston GW, Odling-smee GW, et al. Bleeding oesophageal varices with long tern follow up. Arch Dis Child 1984;59:336-34

7. Zarger SA, Jarid G, Khan BA. Endoscopic ligation compared with sclerotherapy for bleeding varices in children with extra-hepatic portal venous obstruction. Hepatology 2002;36(3):666-667. [http:// dx.doi.org/10.1053/jhep.2002.352788

8. Hill ID, Bowie MD. Endoscopic sclerotherapy for control of bleeding varices in children. Am Gastroenterol 1991;86(4):472-476

9. Itha S, Yaccha SK. Endoscopic outcome beyond oesophageal variceal eradication in children with extrahepatic portal venous obstruction. J Pediatr Gastroenterol Nutr 2006;42(2):1996-2000. [http:// dx.doi.org/10.1097/01.mpg.0000189351.55666.45

10. Zarger SA, Yatoo GN, Javid G. Fifteen year follow up of endoscopic injection sclerotherapy in children with extrahepatic portal venous obstruction. J Gastroenterol Hepatol 2004;19(2):139-145. [http://dx.doi.org/10111/j.1440-1746.2004.03224.x]

11. Dasgupta R, Roberts E, Superina RA, et al. Effectiveness of Rex shunt in the treatment of porta hypertension. J Pediatr Surg 2006;41:108-112. [http://dx.doi.org/10.1016/j.jpedsurg.2005.10.059]

12. Millar AJ, Brown RA, Hill ID. Fundal pile: Bleeding gastric varices. J Pediatr Surg 1992;27(3):419. [http://dx.doi.org/10.1016/0022-3468(91)90015-L]

13. Zarger SA, Javid G, Khan BA, et al. Endoscopic ligation compared with sclerotherapy for bleeding oesophageal varices in children with extrahepatic portal venous obstruction. Hepatology 2002;36(3):666-672. [http://dx.doi.org/10.1053/jhep.2002.35278]

14. Sarin SK, Govil A, Jain AK et al. Sclerotherapy versus variceal band ligation for oesophageal varices: influence on gastropathy, gastric varices and variceal recurrences. J Hepatol 1997;26(4):826-832. [http://dx.doi.org/10.1016/50168-8278(97)80248-615]

15. Duche M, Habes D, Roulleau P, et al. Prophylactic endoscopic sclerotherapy of large osephagogastric varices in infants with biliary atresia. Gastroenterol Endosc 2008;67(4):732-737. [http:/ dx.doi.org/10.1016/j.gie.2007.11.005

16. Thapa BR, Mehta S. Endoscopic sclerotherapy of esophageal varices in infants and children. J Pediat Gastroenterol Nutr 1990;10:430-434

17. Paquet KJ. Ten year experience with paravariceal injection sclerotherapy of oesophageal varices in children. J Pediatr Surg 1985;20:109-112.

Accepted 25 September 2012 